\title{
Les membranes en lipotransformation : bilan, résultats, perspectives
}

\author{
Michel PARMENTIER \\ Sandrine GUILLEMIN \\ Reine BARBAR \\ Michel LINDER \\ Jacques FANNI
}

\author{
Laboratoire de science et génie alimentaires, \\ ENSAIA, INPL, \\ 2 avenue de la Forêt de Haye, \\ F-54500Vandoeuvre les Nancy \\ $<$ Michel.Parmentier@ensaia.inpl-nancy.fr>
}

\begin{abstract}
Membrane separative techniques as candidates for alternative "green" processing of Oils and Fats present several advantages like energy saving, friendliness to environment and quality preservation of the final products. However, despite the considerable number of research programs carried out on several steps of the processing, including refining, desolvantisation and fractionation, no significant industrial development has been observed. Pointing out the lack of fundamental understanding of the mechanisms that rule the separations in hydrophobic media, a renewed approach of the membrane partition based on the relative hydrophobicity of lipids molecules vs barrier materials has been set up. Starting from an electronic analogy, a hydrophobic/hydrophilic valve has been designed by using a polar molecules coating on a porous material surface. The experimental realisation based on a two-compartments cell allowed to obtain preliminary results on the separation of the oily and water phases of an emulsion, that is a very promising result.
\end{abstract}

Key words : membrane, emulsion, hydrophobic/hydrophilic value

terme générique regroupant les systèmes construits autour de milieux poreux minéraux ou organiques organisés suivant des géométries diverses permettant de résoudre les problèmes ardus de débit de perméation, de sélectivité, et de tenue à la corrosion chimique et physique. Historiquement, la première utilisation industrielle lourde de tels milieux de séparation a été mise en œuvre dans les années 1945-50 par I'industrie nucléaire, confrontée au difficile problème de la séparation isotopique ${ }^{235} \mathrm{U} v \mathrm{vs}^{238} \mathrm{U}$. L'une de techniques utilisées, l'effusion gazeuse (figure 2), fait appel à une technique membranaire. Le passage du composé gazeux ${ }^{235} \mathrm{UF}_{6}+{ }^{238} \mathrm{UF}_{6}$ à travers un milieu poreux de type céramique présente une vitesse qui est inversement proportionnelle à la racine de la masse de la particule, ce qui a pour effet, après passage à travers des milliers de cellules $d^{\prime}$ aboutir à un enrichissement du perméat en composé « léger » ${ }^{235} \mathrm{UF}_{6}$.

La technique évoluera ensuite très rapidement et l'industrie naissante des membranes va trouver dans l'industrie alimentaire les conditions d'un développement extraordinaire : la classification des techniques en fonction du seuil de coupure des membranes et des types d'application (micro-, ultra- puis nanofiltration) a permis de multiplier les utilisations dans les problèmes de filtration classique de milieux turbides mais également dans le cas des dispersions protéiques et des solutions chargées. D'où la pertinence des applications des membranes dans l'industrie laitière et celle des boissons.

\section{Techniques membranaires et procédés lipidiques}

Dans le cas des huiles et graisses, nombreux sont les problèmes de séparation qui $a$ priori auraient dû trouver une réponse membranaire, c'est pourquoi le premier brevet américain déposé par Sen Gupta en 1977, Process for refining crude glyceride oils by membrane filtration [1], a soulevé un réel engouement de la recherche. Des équipes importantes se sont alors intéressées à l'extension de la technique dans toutes les étapes de la transformation des huiles et tout particulièrement du raffinage. Le tableau 1 donne une synthèse de tous ces essais publiés, qui ne font d'ailleurs pas état des nombreux autres tests réalisés en industrie.

Les recherches les plus prometteuses ont concerné le dégommage, la désolvantisation, la désacidification et, plus récemment, le fractionnement. Les techniques mobilisées différaient assez peu pour ce qui concerne le montage, mais demandaient la mise en œuvre de membranes particulières, si bien que l'on peut dire que toutes les membranes existantes sur le marché, toutes les astuces de montage possibles ont été mises en œuvre. À titre d'exemple, la figure 3 montre la technique utilisée par $S$. Koseoglu en 1991 [3] sur le recyclage de l'hexane.

Dès 1995, FP Cupperus et TP Derksen [4] soulignent l'absence de développement malgré l'impressionnante liste de travaux qui sont repris dans le tableau 1. Le même constat est
La seconde évolution a concerné les couches filtrantes, avec l'apparition des membranes, 


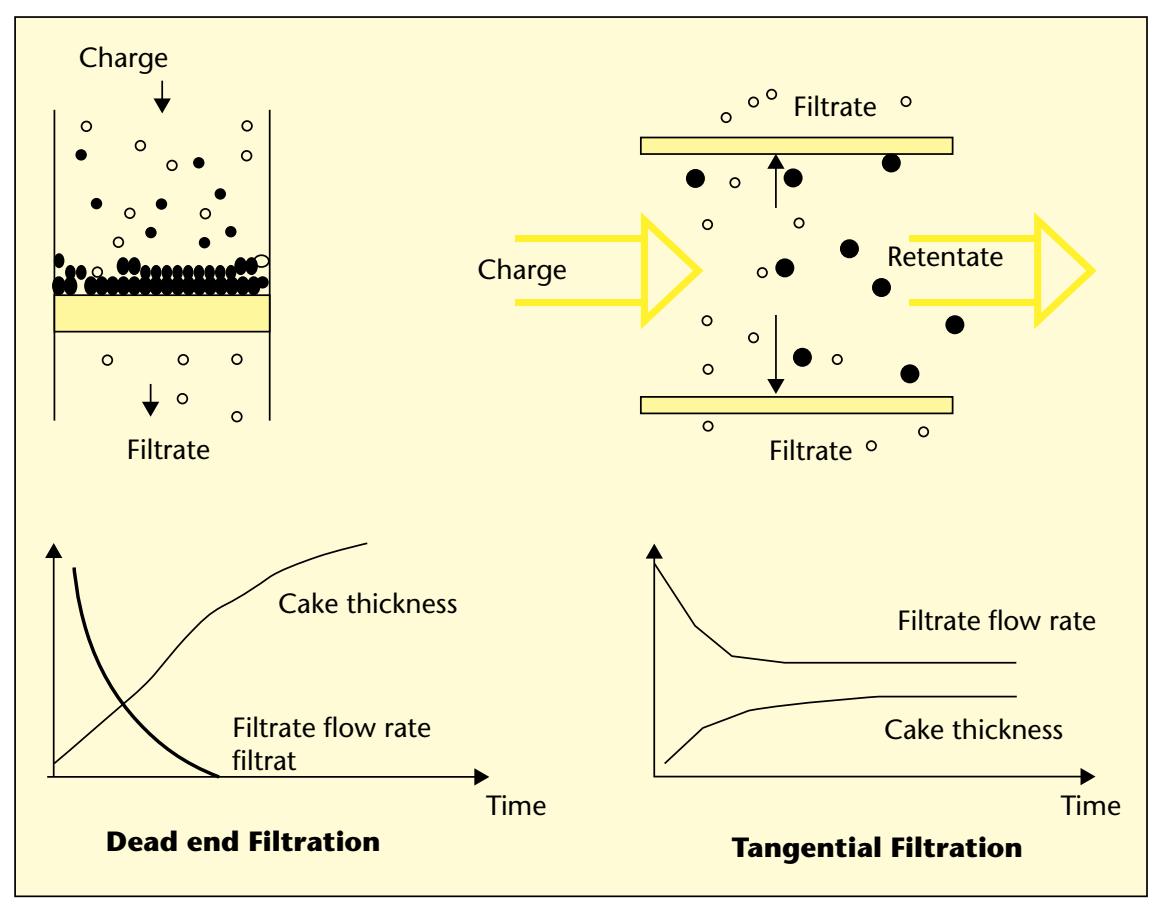

Figure 1. Filtration frontale et filtration tangentielle : montage, débits et couche de polarisation ou gâteau (El Ama, 1998).

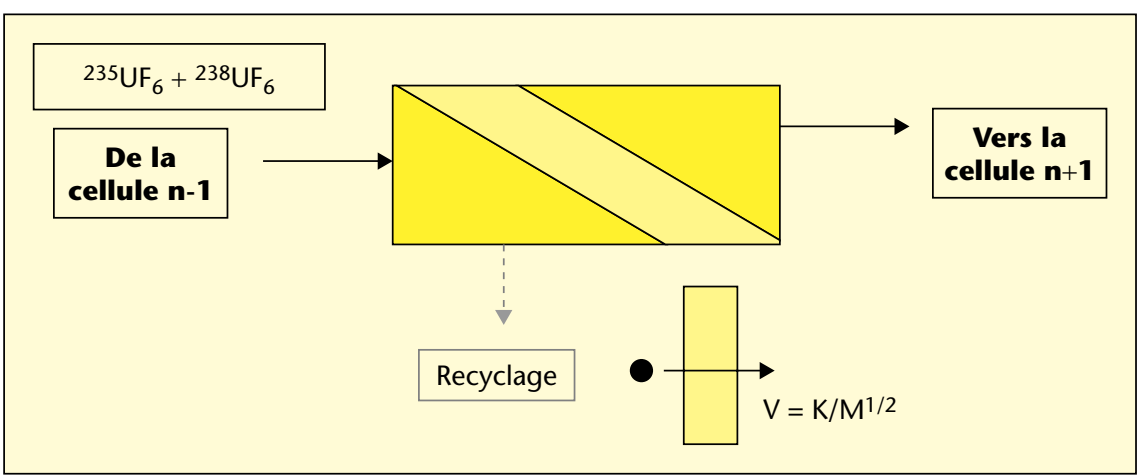

Figure 2. Séparation des isotopes 235 et 238 par effusion gazeuse du gaz UF 6 à travers un milieu poreux.

publié par tous les groupes de recherche actifs aux États-Unis (SS Koseoglu [5] et M Cheryan [6]), au Japon (M Nakajima [7]) et en France (Graille et Pioch [8] à Montpellier, Parmentier et Fanni à Nancy [9]).

En dépit de bilans énergétiques et environnementaux favorables, la technique s'est heurtée à des problèmes aigus de colmatage (entraînant la chute du débit après des temps de filtration insuffisamment longs), d'une faible sélectivité qui entraîne l'obtention de fractions non complètement séparées, et finalement de tenue des systèmes membranaires dans des milieux particulièrement agressifs chimiquement. L'essentiel du problème réside dans le fait que montages et matériaux utilisés en lipo-
Koseoglu en 1991 [10] et fortement orientées sur l'économie d'énergie réalisable aux ÉtatsUnis via l'adoption du raffinage membranaire, les arguments n'ont pas convaincu les opérateurs.

\section{Mécanismes et modèles de séparation membranaires adaptés aux milieux hydrophobes}

Contribuer à progresser dans ce domaine a nécessité de reprendre les travaux fondamentaux sur l'organisation des milieux hydrophobes, dans un premier temps sur des milieux modèles puis sur des huiles et graisses naturelles, autrement plus complexes. Tout d'abord, il convient de sortir de toute analogie entre milieux hydrophiles (solutions dans l'eau de macromolécules, polymères, molécules simples et ions) et milieux hydrophobes constitués à plus de $90 \%$ de molécules glycéridiques monodisperses solubles dans les solvants organiques (figure 4). Si I'on y regarde de plus près, les milieux lipidiques naturels ne sont jamais des phases homogènes : ils contiennent toute une série de structures plus ou moins organisées autour de certaines impuretés structurantes, essentiellement l'eau, toujours présente, et les lipides polaires. L'association des deux donne de multiples superstructures dont les tailles sont très variables (micelles, lamelles, cristaux liquides...) À ces hétérostructures il faut ajouter les associations moléculaires de triglycérides qui sont dépendantes de leur passé thermique. II s'agit d'agrégats moléculaires plus ou moins importants en fonction de la température, c'est-à-dire de l'écart de température entre l'état d'observation et le point de fusion. Ces agrégats sont constitués de molécules orientées conformément à leurs formes cristallines passées. De ces deux remarques découlent deux types de mécanismes possibles de séparation conduisant à un fractionnement sur membrane : le premier fait appel à l'organisation micellaire, hypothèse posée simultanément et largement développée en commun avec l'équipe du Cirad à Montpellier (Larguèze et al., 1995) [11] à l'occasion de travaux sur le raffinage (figure 5). II est fondé sur la filtrabilité de structures organisées autour des impuretés présentes en particulier dans une huile brute, à cause du contenu en lipides polaires (acides gras et glycérides partiels, phospholipides) qui $s^{\prime}$ organisent en micelles avec la participation de l'eau présente.

De même sont filtrables les structures précristallines provenant des agrégats moléculaires rappelant la cristallisation des triglycérides, comme l'indique la figure 6 . Cette hypothèse que nous avons développée en particulier sur le modèle MGLA (matière grasse laitière anhy- 
Tableau 1. Synthèse des principaux essais de techniques membranaires dans le domaine des huiles, d'après Parmentier et al., 2002 [2].

\begin{tabular}{|c|c|c|c|c|c|c|}
\hline Séquence & Huile & Membrane & Config. & Solvant & Auteurs & Année \\
\hline \multirow{5}{*}{$\begin{array}{l}\text { Élimination et } \\
\text { récupération du solvant } \\
\text { Dégommage }\end{array}$} & Coton & PA & RO & Éthanol & Kuk et al. & 1989 \\
\hline & & Polymère Fluoré & $\mathrm{NF}$ & Hexane, Isopropanol & Koseoglu et al. & 1990 \\
\hline & Soja & $\mathrm{PI}$ & UF & Hexane & Iwama & 1986,87 \\
\hline & & & & & Miki et al. & 1988 \\
\hline & & & & & Sen Gupta & 1986 \\
\hline \multirow[t]{4}{*}{ Séparation } & Tournesol & CA & $\mathrm{RO} / \mathrm{NF}$ & Ethanol & Kuk et al. & 1992 \\
\hline & Soja & $\mathrm{PI}$ & UF & Hexane & Keurentjes et al. & 1992 \\
\hline & Soja & PAN & UF & Isopropanol & Sahashi et al. & 1993 \\
\hline & Poisson & $\mathrm{PI}$ & UF & Ethanol & & \\
\hline Décirage & Tournesol & Polym. Synth. & MF & Sans & Chayamizu and Kikuchi & 1988 \\
\hline \multirow{2}{*}{ Décoloration } & Tournesol & $\mathrm{PI}$ & MF & Hexane / sans & Reddy et al. & 1996 \\
\hline & Soja & $\mathrm{PE}$ & MF & Sans & Vaira and Koseoglu & 1993 \\
\hline $\begin{array}{l}\text { Récupération de } \\
\text { Catalyseurs }\end{array}$ & Soja & Céramique & UF & Sans & Koseoglu and Vavra & 1993 \\
\hline Récupération de métaux & Soja & Cellulose & dialyse & $\mathrm{NH} 3$ & Keurentjes et al. & 1990 \\
\hline Blanchiment & Toutes huiles & Céramiques & UF & & Cheryan et al. & 1994 \\
\hline Traitement des eaux usées & Toutes huiles & Céramiques & $\mathrm{RO}, \mathrm{UF}, \mathrm{MF}$ & Sans & Koseoglu & 1991 \\
\hline
\end{tabular}

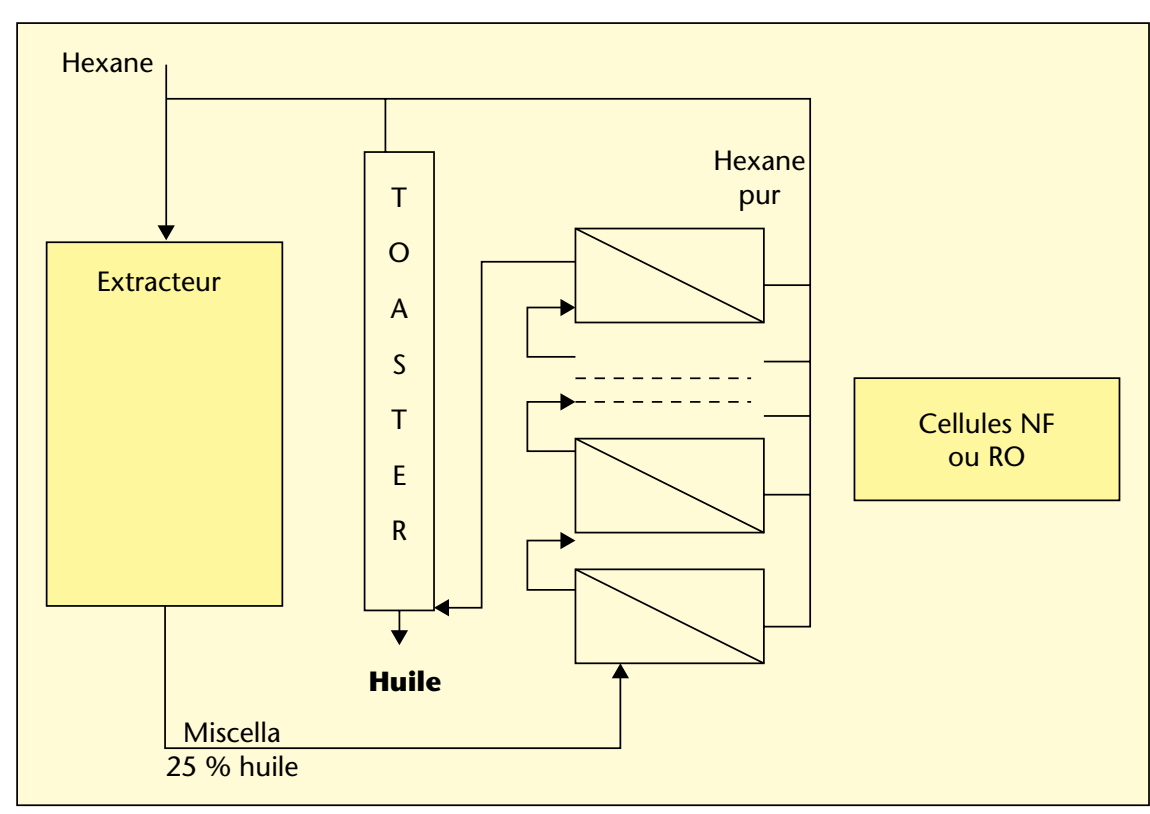

Figure 3. Procédé de désolvantisation et recyclage de I'hexane (Koseoglu, 1991).

dre), a donné des résultats intéressants en laboratoire, mais décevants en configuration industrielle (figure 7).

L'hypothèse était qu'un mélange de triglycérides liquide proche de son point de fusion n'est pas un système homogène : des structures qui rappellent l'organisation de l'état solide continuent d'exister dans une gamme de température de quelques degrés au-dessus du point de fusion. Ces systèmes «micellaires » présents dans le liquide peuvent rendre possible une filtration, car ils augmentent artificiellement la dispersion de masse des agrégats, donc la filtrabilité. De tels systèmes ont de grandes chances d'exister dans les huiles et graisses naturel- les, car ces matériaux biologiques contiennent un grand nombre d'impuretés qui sont autant de produits amphiphiles possédant un rôle structurant au sein d'un milieu qui n'est qu'en apparence homogène.

Le deuxième mécanisme possible met en œuvre les interactions molécules - couche filtrante. Ces interactions entre les triglycérides et certains oxydes métalliques, comme $\mathrm{Al}_{2} \mathrm{O}_{3}$, $\mathrm{SiO}_{2}, \mathrm{TiO}_{2} \ldots$, ont été très peu décrites dans la littérature. C'est donc une recherche « d'amont» que nous avons mis en œuvre sur un montage simplifié de percolation frontale par simple gravité [12]. La composition triglycéridique des rétentats et filtrats, ainsi que celle de la fraction de l'huile adsorbée sur le support divergent, ce qui montre qu'une discrimination se produit sur la couche poreuse (figure 8). Celle-ci est fonction de I'hydrophobicité des molécules, laquelle étant liée à l'insaturation, l'est donc finalement aux points de fusion. Ces résultats montrent que la partition est globalement dépendante de la nature chimique du support filtrant, et de l'hydrophobicité relative des molécules triglycéridiques et de la couche d'oxydes.

Confirmés sur une huile très insaturée (huile de pépins de raisin), ces essais mettent en évidence l'influence de l'insaturation dans ce type de séparation. En effet, les triglycérides ont la propriété d'adapter leur configuration stéréochimique à leur environnement, et tout particulièrement lorsqu'ils se placent à une interface, ce qui est le cas au voisinage d'une membrane : contre une surface hydrophobe, ils adoptent la configuration diapason et les chaînes grasses peuvent ainsi entrer en interaction de Van der Waals avec le support hydrophobe. Inversement, au contact d'une surface polaire, certaines de ces molécules peuvent prendre la configuration trident de façon à ce que les oxygènes des liaisons esters puissent engager des liaisons hydrogène avec le support (figure 9). Des mécanismes de fractionnement sont donc possibles, suivant le type de couche filtrante utilisée : une surface filtrante hydrophile tendra à retenir les molécules en conformation diapason et laissera traverser celles qui sont capables d'adopter la configuration trident, ce qui dépend essentiellement de deux paramètres structuraux : la longueur des chaînes et leur insaturation [12]. La situation finale sera exactement inversée dans le cas d'une couche filtrante hydrophobe. 


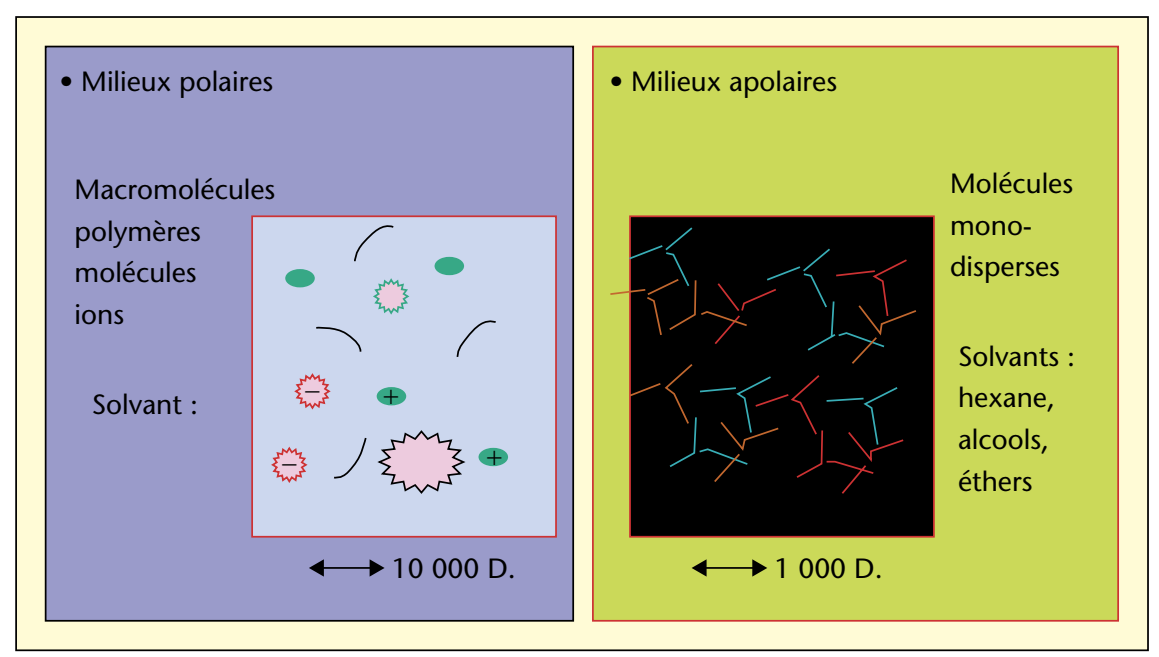

Figure 4. Structure des milieux hydrophiles et hydrophobes (Parmentier, 1999).

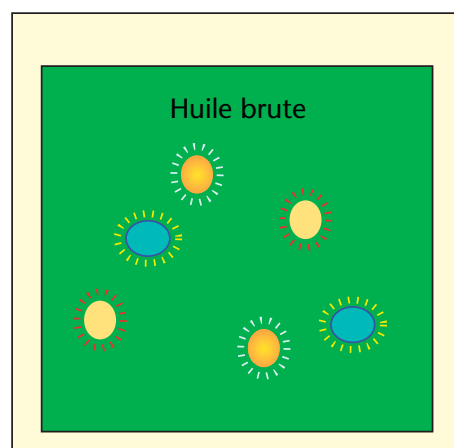

$[\mathrm{P}]>\mathbf{5 0 0} \mathbf{p p m}$
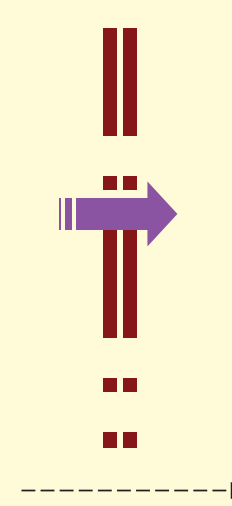

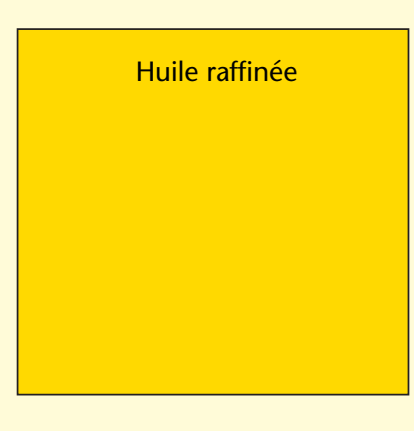

$[\mathrm{P}]<5$ ppm
Figure 5. Raffinage d'huile de tournesol par microfiltration sur membrane hydrophobe, MF (Largueze et al., 1995, [11]).

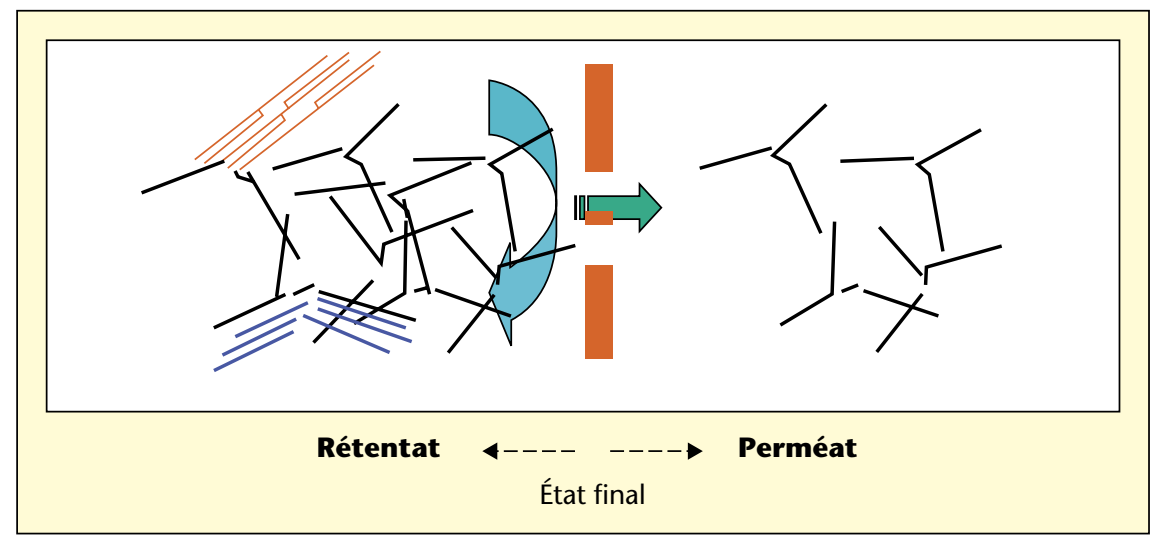

Figure 6. Modèle de transition solide-liquide des triglycérides et faisabilité d'une séparation sur membrane.
Les travaux de P Lonchampt en 2001 [13] dont le but était d'aboutir à une quantification des forces mises en jeu dans les interactions en milieu hydrophobe grâce à une étude menée sur des systèmes modèles huile - supports simplifiés à l'extrême afin de permettre le calcul. Ce travail a permis de préciser les relations structurales qui s'établissent entre les triglycérides et des silices dont l'hydrophobicité a pu être modifiée de façon quasi continue. La mobilisation de méthodes physiques comme la RMN basse résolution, l'infrarouge à transformée de Fourier et la calorimétrie différentielle ou isotherme a permis de préciser la nature chimique des interactions, et tout particulièrement l'implication de la liaison ester dans la couche limite en contact avec le support, ce qui constitue un apport majeur au problème posé, confirmé d'ailleurs récemment par Hafidi et al., 2004 [14].

Par ailleurs, certaines configurations expérimentales conduisant à des mélanges de molécules grasses à points de fusion bien séparés peuvent être fractionnés sur membrane hydrophobe, parfaitement compatible avec de tels milieux. C'est le cas par exemple du fractionnement d'une huile de poisson réalisée après déstructuration par une lipase. Les acides gras et glycérides partiels formés ont des propriétés de fusion bien différenciées, ce qui conduit à un fractionnement efficace sur membrane, donnant un rétentat solide et un perméat liquide à la température ambiante de $20^{\circ} \mathrm{C}$ (figure 10). Toutefois, il convient de noter qu'il s'agit-là de mélanges non directement comestibles car contenant une grande quantité d'acides gras libres.

Ces mécanismes - forcément schématiques ont été mis en œuvre pour fractionner la MGLA, dans la mesure où il est clair aujourd'hui que l'interaction entre le matériau support et les triglycérides est fortement dépendante de I'hydrophobicité relative des deux types de molécules qui interagissent. Or, la polarité des triglycérides varie, d'une part avec la longueur des chaînes grasses, mais aussi et surtout avec leur insaturation, ce qui se traduit macroscopiquement par une différence de point de fusion. Un fractionnement est donc possible, mais il ne peut conduire aux mêmes types de phases extrêmes que le cryofractionnement à multiples étapes. En effet, il n'est pas possible de pousser la sélectivité à ce niveau, en tout cas à ce stade de la recherche, qui doit s'orienter vers des matériaux-barrière de quatrième génération, capables d'engager des interactions moléculaires spécifiques [15]. 


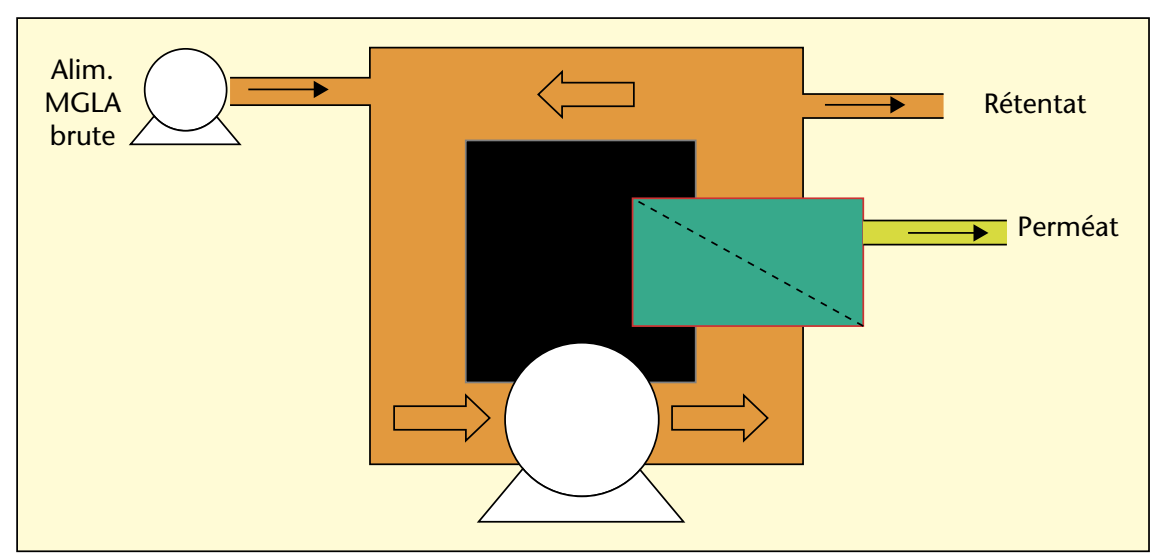

Figure 7. Montage de filtration tangentielle de MGLA : une boucle de rétentat qui s'enrichit en triglycérides à haut points de fusion au fur et à mesure du soutirage du perméat.

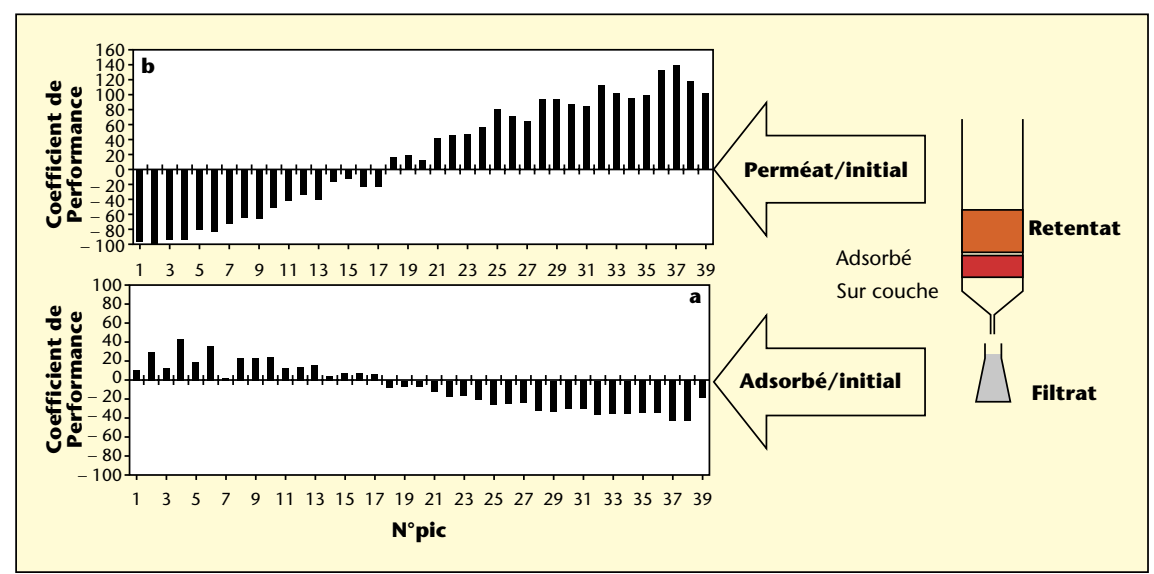

Figure 8. Filtration frontale par gravité à $45^{\circ} \mathrm{C}$ de MGLA sur membrane en silicagel. Rapport de concentrations des triglycérides perméat/initial et huile adsorbée/initial.

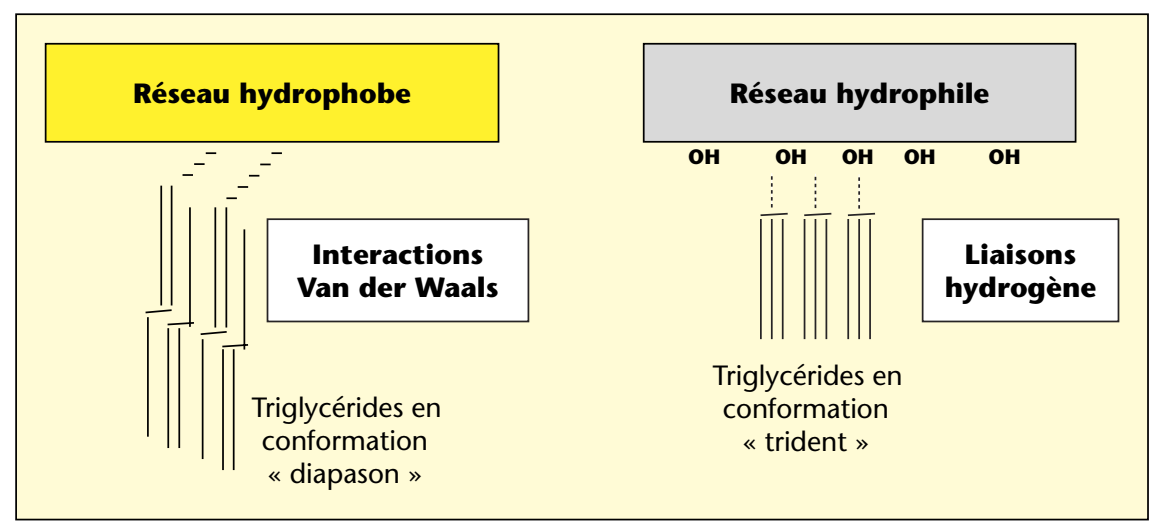

Figure 9. Modèle d'interaction triglycéride-membrane (d'après [12]).

\section{Approche actuelle : la séparation sur membrane des constituants d'une émulsion}

Obtenir une émulsion alimentaire stable a été I'un des sujets de recherche les plus travaillés ces dernières années, mais le problème inverse peut être quelque fois beaucoup plus ardu encore : séparer les constituants d'une émulsion naturelle ou technologique, c'est-à-dire réobtenir d'un côté la phase grasse et de l'autre la phase aqueuse. Certaines configurations industrielles nécessitent justement ce type de " démixion », comme par exemple en traitement des effluents ou en extraction aqueuse d'huiles végétales. C'est dans ce but que nous avons développé un modèle original : la valve hydrophile/hydrophobe: sur la base d'une analogie avec la diode électronique, il est possible d'imaginer un milieu poreux orienté de telle façon que la compatibilité milieu/molécules permette le passage des molécules dans un sens seulement en fonction de leurs propriétés de surface. Cette aptitude est obtenue dans la diode électronique par dopage d'un cristal composé d'ions tétravalents avec, d'un côté, des ions pentavalents et, de l'autre, par des ions trivalents, créant du même coup un déséquilibre électronique qui polarise le cristal.

Notre hypothèse pour réaliser un dispositif similaire avec une orientation des surfaces est fondée sur le tapissage d'une face par une molécule amphipolaire orientée de telle sorte que la surface soit polaire d'un côté, et de l'autre de la même molécule orientée côté apolaire à l'extérieur (figure 11). Alimentée dans un sens par un mélange de molécules polaires et apolaires (ou émulsion), seules les molécules compatibles avec la surface en regard peuvent pénétrer dans la membrane poreuse. Les autres sont exclues et restent donc dans la charge. On observe la situation inverse si on alimente dans l'autre sens.

Une telle valve interfaciale peut alors fonctionner comme système de séparation des constituants hydrophiles et hydrophobes d'un milieu complexe. Pour continuer l'analogie électronique, considérons le transistor, association de deux diodes tête-bêche PNP ou NPN. L'association de deux valves en opposition permet de réaliser un montage analogue, tel que représenté dans la figure 12. L'alimentation de cette cellule par une émulsion doit conduire à un «tri » moléculaire en fonction des propriétés interfaciales de chacune d'entre elles : les éléments hydrophiles traversent la valve orientée face hydrophile vers le flux tangentiel, alors que les molécules apolaires traversent l'autre. L'état final conduit à une séparation de l'émulsion en ses constituants en fonction de leurs 


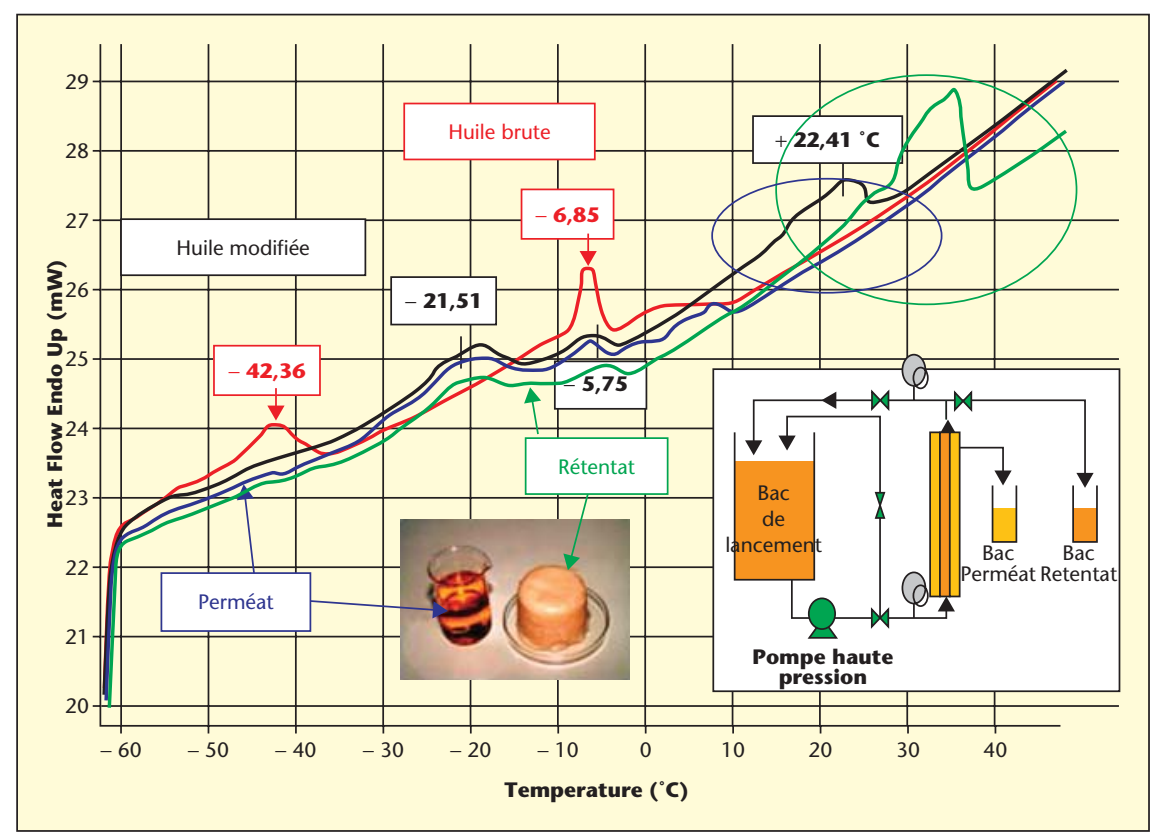

Figure 10. Séparation sur membrane des fractions d'huile de saumon déstructurées par l'action d'une lipase. Visualisation des fractions par les points de fusion en DSC.

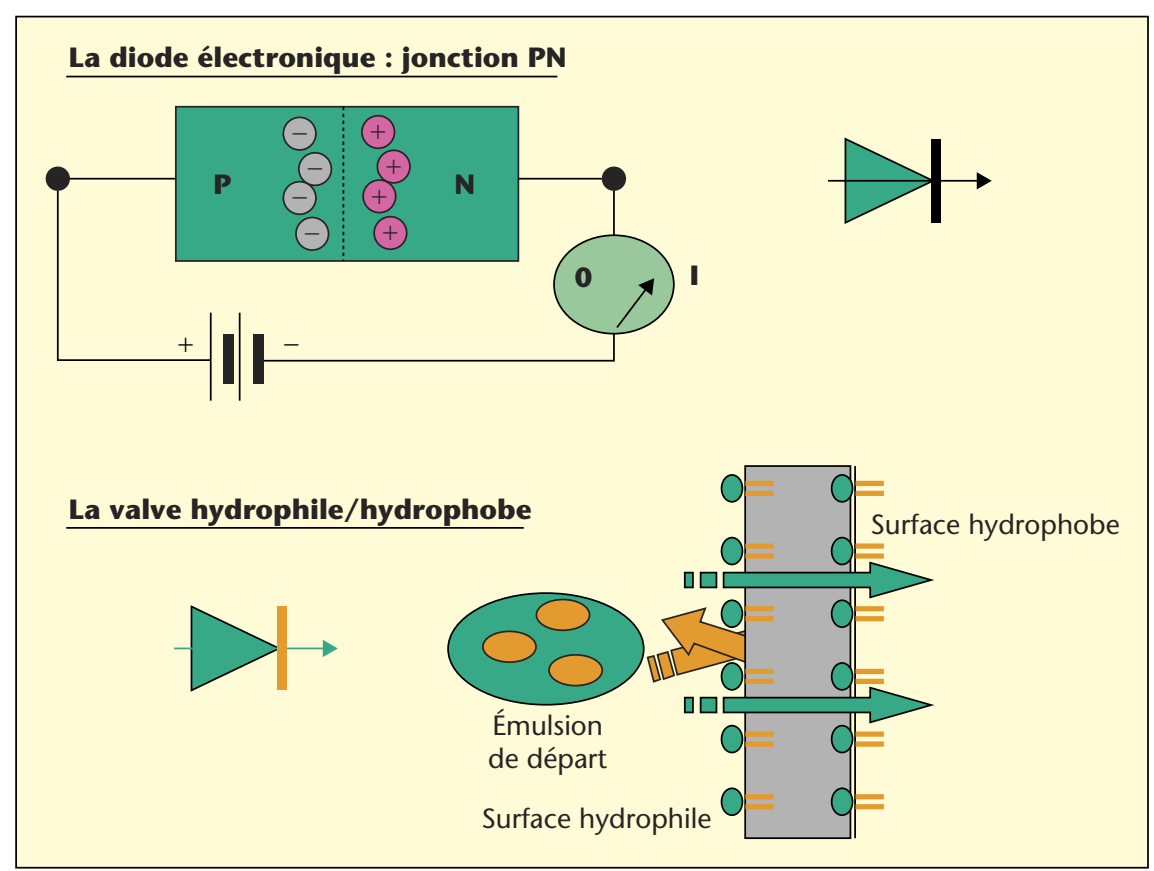

Figure 11. Construction d'une valve hydrophile/hydrophobe sur la base de l'analogie avec la diode électronique.

propriétés donc de leur affinité vis-à-vis des surfaces en regard.

Ainsi une cellule de séparation peut être construite suivant le schéma de la figure 12: une boucle équipée d'une pompe de circulation est alimentée en émulsion fraîche. Le flux tangentiel traverse la cellule composée des deux valves qui déterminent un compartiment hydrophile et un compartiment hydrophobe dans lesquels sont collectées les fractions.

On peut s'approcher de ce montage théorique en utilisant le dispositif simplifié représenté figure 13. L'astuce technique consiste à remplacer la boucle d'alimentation par une chambre circulaire dans laquelle le fluide de charge est mis en mouvement circulaire par un agitateur magnétique. De cette façon, les vecteursvitesse des molécules sont en tout temps tangentiels par rapport aux surfaces actives. Les deux valves sont montées de façon diamétralement opposée si bien que les fractions sont collectées en arrière de chaque valve. Un tel système a été construit et alimenté par une émulsion huile de maïs-eau stabilisée par le sodium-dodécyl-sulfate. Après quatre heures de fonctionnement, en utilisant une légère pression d'azote (inférieure à $1 \mathrm{bar}$ ), on retrouve la phase aqueuse dans le compartiment hydrophile et l'huile de l'autre côté. C'est la première fois, à notre connaissance, qu'une telle séparation est observée sur un système à membrane.

Le potentiel d'application de ces techniques est énorme, puisque si la constitution et la stabilisation des émulsions alimentaires ont fait l'objet d'une puissante recherche depuis les années 1970-80, leur déstabilisation et la libération des constituants sont un problème encore plus difficile à résoudre. Or, les émulsions ne sont pas toujours désirables et, dans de nombreuses configurations industrielles, " casser » l'émulsion est obligatoire, soit pour extraire l'un des constituants, soit pour récupérer la phase aqueuse. Toutefois, il convient de noter que l'essentiel de l'effort de recherche est aujourd'hui tourné vers l'étude de matériaux membranaires nouveaux, adaptés aux milieux hydrophobes. En effet, pour le moment, des membranes commerciales hydrophiles et hydrophobes ont été utilisées, mais leur structure ne correspond pas exactement à la mise en œuvre d'une valve hydrophile ou hydrophobe, telle que nous l'avons imaginée d'un point de vue fondamental. La mise au point de nouveaux matériaux membranaires fournissant une véritable «échelle » d'hydrophobicité est un problème qui nous préoccupe majoritairement aujourd'hui.

En termes d'applications, deux domaines sont particulièrement prometteurs dans ce schéma général : la déstabilisation des émulsions produites lors de l'extraction des huiles végétales par voie aqueuse, et la rupture des émulsions que sont les effluents liquides des huileries afin, $d^{\prime}$ une part, d'en récupérer la phase aqueuse et, d'autre part, de concentrer les polluants gras dans deux buts : diminuer la charge DCO de l'effluent et valoriser le déchet gras dans la filière énergétique ou chimique.

Ces deux types d'applications font actuellement l'objet de recherches actives en collaboration avec la filière oléagineuse française et l'industrie.

Remerciements. Les auteurs remercient vivement I'Onidol (Paris), pour le soutien financier qui permet de poursuivre ces travaux à caractère fondamental. 


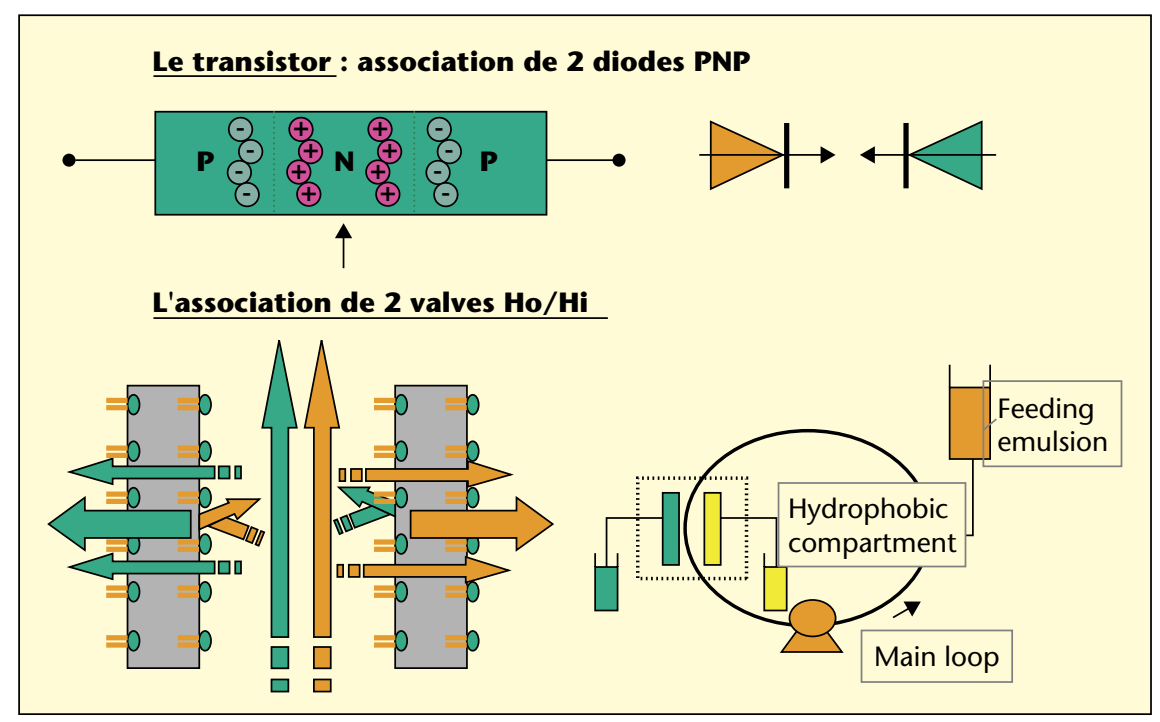

Figure 12. Montage «tête-bêche » de deux valves hydrophile/hydrophobe sur la base de l'analogie avec le transistor (schéma de principe et réalisation expérimentale).

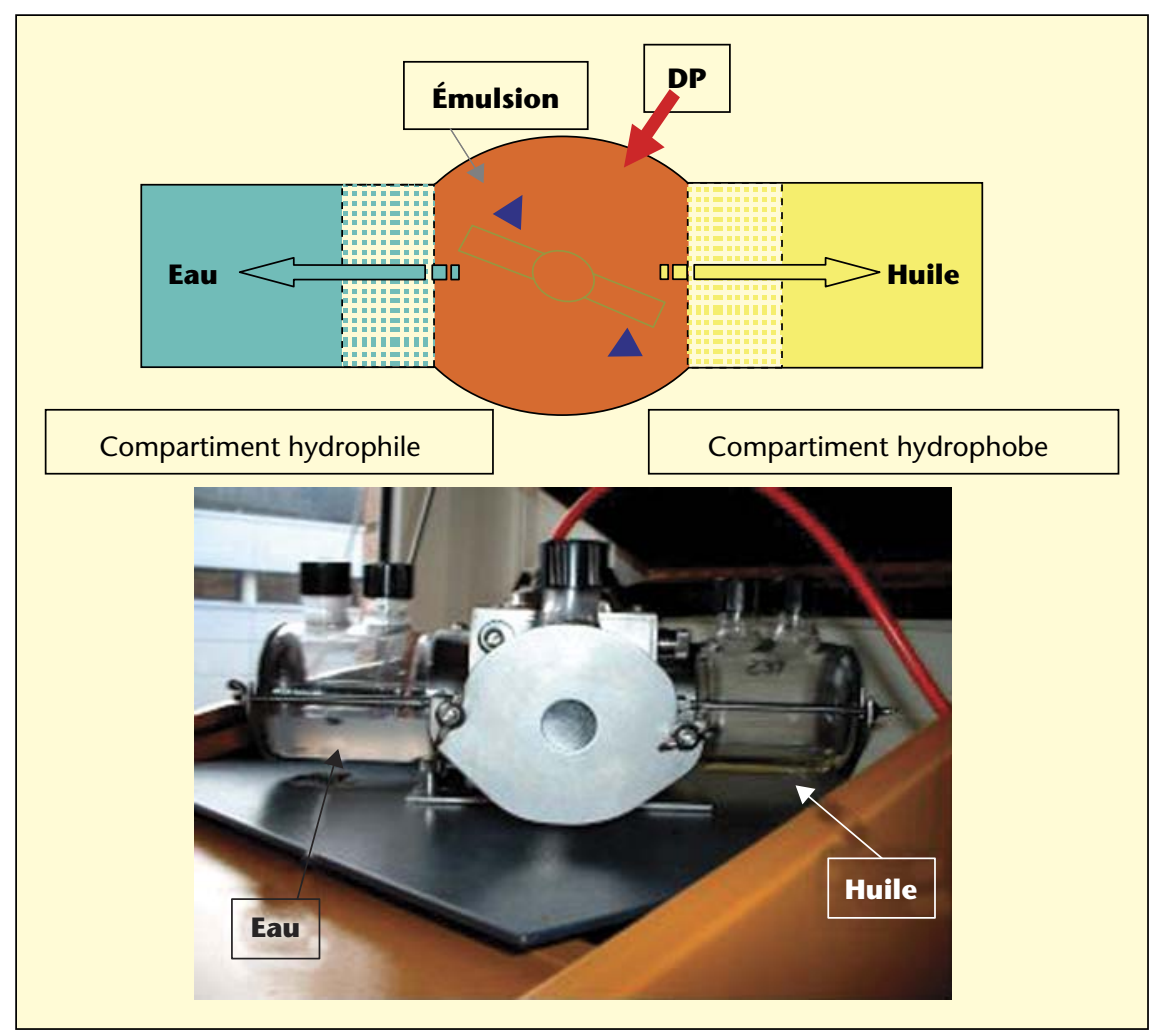

Figure 13. Démixion d'une huile de maiis-eau-SDS par une cellule à deux valves hydrophile/hydrophobe (schéma de principe et réalisation expérimentale).

\section{RÉFÉRENCES}

1. SEN GUPTA AK. Process for refining crude glyceride oils by membrane filtration. US Patent $1977 ; 4(062): 882$.

2. PARMENTIER M, FANNI J, LINDER M. Les technologies membranaires en lipotechnie. In : Lipides et Corps Gras Alimentaires. Paris : Tec \& Doc, Lavoisier, 2002 ; (56 p).

3. KOSEOGLU SS, LAWHON JT, LUSAS EW. Membrane processing of crude vegetable oils : pilot plant scale removal of solvent from oil miscella. J Am Oil Chem Soc $1990 ; 67(5)$ : 31522.

4. CUPPERUS FP, DERKSEN TP. Applications of membrane technology in the processing of vegetable oils and derivatives. Lipid Technol, PJ Barnes \& Ass 1995 : 101-7.

5. KOSEOGLU SS. Membrane Technology for Edible Oil Refinig. Oils \& Fats Int 1991 ; 5 : 16-21.

6. RAMAN LP, RAJAGOPALAN N, CHERYANM. Membrane Technology. Oils \& Fats Int 1994 ; $10(6):$ 28-38.

7. SNAPE JB, NAKAJIMA M. Processing of agricultural fats and oils using membrane technology. J Food Eng 1996 ; 30 : 1-41.

8. PIOCH D, LARGUEZE $C$. Les techniques membranaires en lipotechnie. OCL $1995 ; 2$ : 355-8.

9. PARMENTIER M, FANNI J, LINDER M. Revisiting membrane filtration in oils and fats. Inform $2001 ; 12: 411-8$.

10. KOSEOGLU SS. Membrane technology for edible oil refining. Oils \& Fats International 1991 ; $5: 16-21$.

11. PIOCH D, LARGUÈZE C, GRAILLE J, AJANA H, ROUVIÈRE J. Toward an efficient membrane based process for vegetable oils refining. Ind Crops Prod $1997 ; 7$ : 83-9.

12. EL AMA C, LINDER M, FANNI J, PARMENTIER M. Crossflow filtration of oils on metal oxides. Influence of the hydrophobicity of the layer. Eur I Lipid Sci Technol 2000 ; 1 : 7-14.

13. LONCHAMPT P. Interactions entre triacylglycérols et matériaux poreux : approche thermodynamique et physico-chimique. Thèse de I'INPL, Nancy, France, 2001.

14. HAFIDI A, ANGLARET E, PIOCH D, AJANA H. Diffuse reflectance Fourier transform infrared spectroscopy of vegetable oils components adsorption on inorganic membranes. Eur / Lip Sci Techn $2004 ; 106: 11-21$.

15. PARMENTIER M, FANNI J. Les techniques membranaires, quel avenir dans le domaine des corps gras. OCL $1998 ; 5: 363-6$. 